\title{
ANÁLISE DE NECESSIDADES PARA A ELABORAÇÃO DE ESTRATÉGIAS DE ENSINO E APRENDIZAGEM AOS ALUNOS DE ENGENHARIA DE PRODUÇÃO NA DISCIPLINA DE MARKETING.
}

André Luís Ortiz Pirtouscheg - andrepirtouscheg@ gmail.com

UNESP - Universidade Estadual Paulista, "Júlio de Mesquita Filho", Campus Guaratinguetá

Endereço - Av. Ariberto Pereira da Cunha, 333, Portal das Colinas, Guaratinguetá, S.P., CEP 12.516-410.

Rubens Alves Dias - rubens.alves@unesp.br

UNESP - Universidade Estadual Paulista, "Júlio de Mesquita Filho", Campus Guaratinguetá Endereço - Av. Ariberto Pereira da Cunha, 333, Portal das Colinas, Guaratinguetá, S.P., CEP 12.516-410.

Resumo: Este trabalho apresenta uma pesquisa-ação sobre possíveis formas de ensino e aprendizagem aplicadas ao curso de Engenharia de Produção. A pesquisa e o método foram desenvolvidos e aplicados aos alunos do quarto ano de engenharia de produção na disciplina de Marketing, sendo importante ressaltar que as pesquisas foram submetidas e aprovadas por um comitê de ética e está cadastrada na Plataforma Brasil. O objetivo desta pesquisa consiste em desenvolver abordagens educacionais que atendam às necessidades dos alunos envolvidos, visando que sejam as mais adequadas aos seus estilos de aprendizagem. As estratégias definidas tiveram como base a aplicação dos questionários de Index of Learning Styles (ILS), que avaliam as preferências em quatro escalas do modelo de estilo de aprendizagem oferecendo insights sobre ensino e aprendizagem, baseado em psicologia educacional e no ensino da engenharia; foi utilizada também a Analytic Hierarchy Process (AHP) com a intenção de criar uma escala hierárquica de prioridades das informações colhidas nas pesquisas com relação aos métodos analisados para a definição das estratégias, com base no referencial teórico educacional sociointeracionista de Vygotsky. Os resultados obtidos até o momento apontam para a questão da necessidade de se definir de forma científica os meios de ensino e aprendizagem, os quais atendam aos alunos em questão; estas estratégias já foram devidamente definidas estando em fase de aplicação, cabendo em uma segunda etapa a avaliação das percepções dos alunos em relação às estratégias utilizadas para a verificação de possíveis melhorias, para isso pretendese aplicar o método ou diagrama de Kano.

Palavras-chave: Estratégia. Ensino e Aprendizagem. Vygotsky. Engenharia.

\section{INTRODUÇÃO}

O crescimento do número de alunos tendo acesso ao ensino superior no Brasil e no mundo dobrou de 100 milhões para 207 milhões entre 2000 e 2014 (UNESCO, 2017). Este fenômeno mundial vem impactando no surgimento de novas instituições de ensino e demanda de professores que muitas vezes não estão totalmente preparados para assumir uma sala de aula em sua integralidade. No caso dos cursos de engenharia, engenheiros se tornam professores, em boa parte, sem os conhecimentos didático e pedagógico necessários para lecionar, este fato está ligado à ausência de formação inicial ou licenciatura destinada à engenharia (RAMMAZZINA FILHO et al., 2014). Por outro lado, o perfil do aluno também vem mudando os jovens ou alunos da geração Y, sendo estes mais impulsivos, destemidos, multitarefas e não imaginam um mundo 
sem tecnologia e comunicação instantânea, atribuindo aos mesmos um ritmo mais acelerado (OLIVEIRA, 2009).

A necessidade de buscar formas de ensino e aprendizagem que atendam a este cenário vem sendo suprida pelas nomeadas metodologias ativas, as quais são replicadas em sala de aula muitas vezes não se preocupando com a real necessidade de adequação para atender às características de um determinado de um grupo de alunos. Na opinião de Mazzioni (2013, p. 95), "a forma como o professor planeja suas atividades de sala de aula é determinante para que os alunos reajam com maior ou menor interesse e contribui com o andamento da aula".

Este trabalho demonstra uma possível abordagem para desenvolver estratégias de formação profissional que atendam às necessidades dos alunos de Engenharia de Produção na disciplina de Marketing e se sejam mais adequadas aos seus estilos de aprendizagem. O Artigo busca responder a questão relativa a importância de elaborar estratégias de ensino e aprendizagem com foco no aluno e qual o impacto no processo de preparação dos discentes.

O processo de mapeamento dos estilos de aprendizagem predominante aos alunos, por meio da aplicação do questionário de Index of Learning Styles (ILS), o qual demonstrou as tendências aprendizagem com base nas características cognitivas dos alunos. A escala de prioridade para definição das abordagens educacionais mais adequadas aos alunos foi realizada com base na Analytic Hierarchy Process (AHP) e por fim foi desenvolvido e aplicado um plano estratégico de ensino e aprendizagem. O trabalho ainda contempla em uma próxima etapa a medição da satisfação dos alunos e, para isso, pretende-se aplicar o modelo ou diagrama de Kano. A adoção de um referencial teórico educacional, em particular o sociointeracionismo de Vygotsky, também faz parte da base estrutural deste trabalho, fornecendo subsídios teóricos e base conceitual para a elaboração das estratégias educacionais.

Até o momento foram realizadas as etapas de aplicação da pesquisa e tabulação dos resultados do questionário, a definição da hierarquia das necessidades dos alunos, o desenvolvimento das abordagens a serem utilizadas em sala de aula e o projeto encontra-se na fase de aplicação aos alunos. Mesmo ainda não finalizado em sua totalidade o projeto apresenta uma contribuição demonstrando um caminho de forma científica e validada para construção e elaboração de estratégias de ensino e aprendizagem com foco nas necessidades dos alunos.

\section{MÉTODO}

O presente trabalho trata-se de uma pesquisa-ação realizada no Centro Universitário Salesiano de São Paulo, Campus São Joaquim, na cidade de Lorena, São Paulo, aplicada somente aos alunos matriculados no quarto ano do curso de Engenharia de Produção e na disciplina de Marketing. Com relação à classificação da pesquisa ela se apresenta quanto à natureza aplicada, pois objetiva gerar conhecimentos para aplicação prática dirigida à solução de problemas específicos. Com relação ao objetivo apresenta-se como explicativa a maioria das pesquisas explicativas utiliza o método experimental, "aprofunda o conhecimento da realidade porque explica a razão, o porquê das coisas" (GIL, 2010, p. 28).

Quanto à forma de abordagem, a pesquisa será combinada, isto é, quantitativa e qualitativa. Quanto ao método é uma pesquisa-ação e, nesse tipo de pesquisa, os pesquisadores e os participantes envolvem-se no trabalho de forma cooperativa. A pesquisa-ação não se refere a um simples levantamento de dados ou de relatórios a serem arquivados. Com a pesquisa-ação, os pesquisadores pretendem desempenhar um papel ativo na própria realidade dos fatos observados (GIL, 2010). A pesquisa obedece a um roteiro dividido em etapas como apresentado na Figura 1. 
Figura 1 - Roteiro de Pesquisa

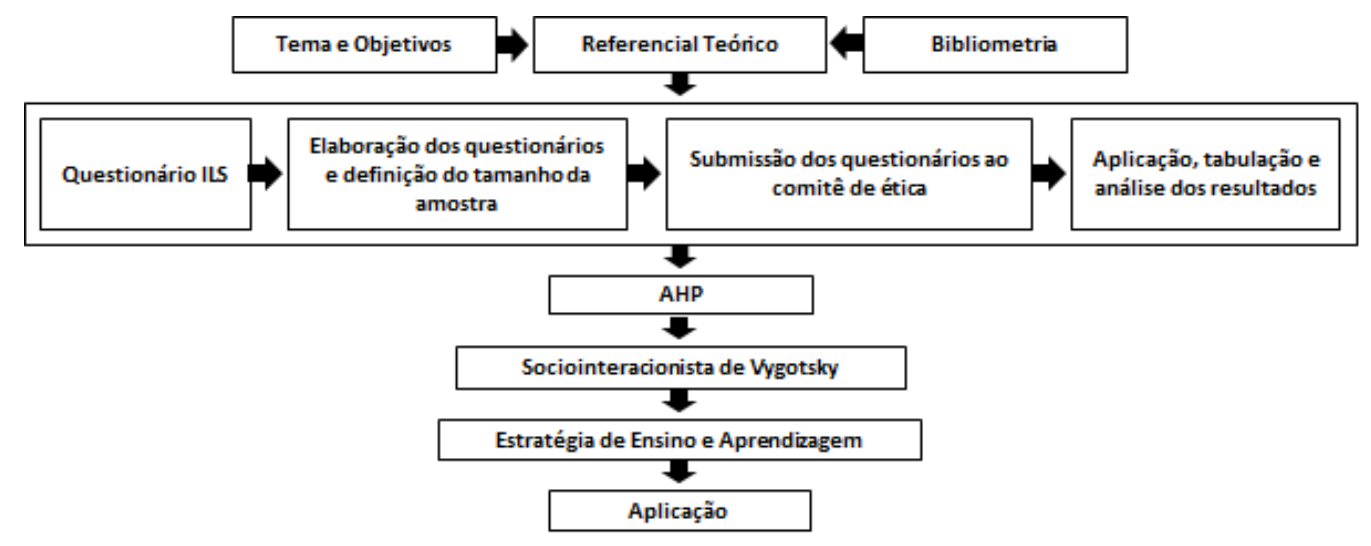

Fonte: Autores

\subsection{Bibliometria}

A bibliometria abrange métodos quantitativos para análise de produções científicas, com o objetivo principal de mensurar um determinado tema a ser abordado. Esta análise de conteúdo busca títulos, palavras-chave, resumos, textos, autores, instituições, autorias, coautorias e referências bibliográficas sobre um determinado foco de estudo (FERREIRA et al., 2015).

Com referência revisão bibliométrica realizada sobre o tema abordado para este projeto por ser vista na Tabela 1 abaixo.

Tabela 1 - Resultado da revisão bibliometrica.

\begin{tabular}{l|c}
\hline $\begin{array}{l}\text { Palavras-chaves para pesquisa em "title- } \\
\text { abstract-keywords" }\end{array}$ & $\begin{array}{l}\text { Resultado na quantidade de "article or } \\
\text { review" }\end{array}$ \\
\hline "learning theory" & 333 \\
\hline "learning styles" & 196 \\
\hline "teaching engineering & 48 \\
\hline "engineering education" & 32 \\
\hline
\end{tabular}

Fonte: Autores

\subsection{Organização da Pesquisa}

As pesquisas foram aplicadas somente aos alunos do $4^{\circ}$ do curso de engenharia do Centro Universitário Salesiano de São Paulo, unidade de Lorena. Os instrumentos de pesquisa, por envolverem seres humanos de forma direta no processo, foram submetidos ao comitê de ética do Centro Universitário de São Paulo e devidamente cadastrado e aprovado na plataforma Brasil sobre o número do comprovante de autorização 143623/2019.

O tamanho da amostra corresponde a 22 alunos, sendo que 21 destes alunos responderam o questionário, o que representa uma margem de erro de 5\% e um nível de confiança de $95 \%$

\subsection{Questionário de Estilo de Aprendizagem}

O instrumento chamado Índice de Estilos de Aprendizagem (Index of Learning Styles - ILS), baseado em auto pontuação, que avalia as preferências em quatro escalas do modelo de estilo de aprendizagem com o objetivo de oferecer alguns insights sobre ensino e aprendizagem (FELDER; SILVERMAN, 1988). 
O Index of Learning Styles - ILS foi sofrendo sucessivas reestruturações, até ser lançado em 1997 na World Wide Web, para uso livre e sem custo associado. A partir do modelo de FelderSilverman relativo aos estilos de aprendizagem, Felder; Soloman (1991), da Universidade da Carolina do Norte, desenvolveram um instrumento denominado por Questionário do Índice de Estilos de Aprendizagem, que classifica os estudantes em quatro das dimensões já anteriormente mencionadas. Este instrumento foi selecionado para utilização, visto que avalia o que a classificação de Felder-Silverman teoriza. Os estudos comprobatórios da sua validade e confiança também contribuíram para a sua utilização. O ILS mede vários componentes da aprendizagem: estratégias de processamento cognitivo, estratégias de regulação, modelos mentais de conhecimento e motivos de aprendizagem (STAVENGA JONG; WIERSTRA; HERMANUSSEN, 2006).

O ILS consiste em quatro escalas, com dois polos e onze escalas. Felder; Spurlin (2005) resumiram as quatro dimensões da seguinte forma:

a) Ativo (esforço ao aprendizado, gosta de trabalhar em grupo) ou Reflexivo (aprende a pensar, prefere trabalhar sozinho ou com um ou dois parceiros);

b) Sensitivo (concreto, prático, voltado para fatos e procedimentos) ou Intuitivo (conceitual, inovador, orientado para teorias e significados subjacentes);

c) Visual (prefere representações visuais do material apresentado, tais como imagens, diagramas e fluxogramas) ou Verbal (prefere escrita e explicações comentadas);

d) Sequencial (processo de pensamento linear, aprende com passos incrementais) ou Global (processo de pensamento holístico).

\subsection{Analytic Hierarchy Process (AHP)}

O método Analytic Hierarchy Process (AHP) busca fornecer aos pesquisadores que enfrentam muitos critérios de avaliação, uma análise estrutural para avaliar os problemas identificar a decisão mais assertiva possível (SAATY; VARGAS, 2006; LAI; LIN, 2015), sendo que sua principal contribuição conceitual ao conhecimento científico consiste na decomposição ou agregação dos elementos (critérios e subcritérios) de um problema por meio de modelagem matemática, com o intuito de constituir uma escala de proporções das prioridades relativas entre os elementos anteriormente mencionados (SAATY; TRAIN, 2007), visando localizar a inconsistência e sugerir o valor ideal para melhorá-la.

O método de pesquisa AHP sistematiza questões e usos complexos em escala de razão e escalas nominais para realizar comparações em pares e estabelece os elementos de uma matriz. Esta forma de pesquisa, geralmente, aplica-se o método de autovetores para calcular os fatores locais e globais e determinar os pesos para indicadores e, após o cálculo, e constituído as fileiras dos indicadores por ordem de peso local e global para indicar a prioridade de importância (LAI; LIN, 2015).

\subsection{O Cognitivismo e o Sociointeracionismo de Vygotsky}

Entende-se por cognitivismo o processo de atribuir significado ao conhecimento por meio da realidade, isto é a partida de experiências sensoriais, representações, pensamentos e lembranças (NUNES; SILVEIRA, 2009). A psicologia cognitiva, trabalha nos processos mentais superiores tem como foco de estudo como as pessoas percebem, lembram e pensam a informação (LEFRANÇOIS, 2015). 


\begin{abstract}
Dentre os conceitos presentes na abordagem cognitivista, aqui são destacadas as teorias desenvolvidas por: Jean Piaget como a epistemologia genética quanto à análise do desenvolvimento do indivíduo e a construção da inteligência; Lev Vygotsky, por intermédio da teoria sociointeracionista, apresentando a mediação pelo parceiro mais capaz na construção do conhecimento operados pelos sistemas simbólicos que organizam os signos em estruturas complexas e articuladas, com base em um conceito histórico-cultural; David Ausubel, o qual contribuiu com o conceito da aprendizagem significativa e a utilização de mapas conceituais como recursos instrucionais; Jerome Bruner com aplicação de questionamentos a fim de entender sobre a mente e a criação de significados (MOREIRA, 2017).
\end{abstract}

Vygotsky era um dos poucos teóricos que entendiam o papel formador da cultura onde o indivíduo é inserido (BRUNER, 1997).

$\mathrm{Na}$ teoria sociointeracionista (também chamada de sociocultural ou histórico-cultural) de Vygotsky, o conceito da zona de desenvolvimento proximal (ZDP) surge do estudo de como o conhecimento já existente, o nível atual de desenvolvimento, pode ser usado para alcançar o próximo nível de conhecimento, o nível potencial, e entre estes dois níveis é que se encontra a ZDP, na qual as estratégias de ensino e aprendizagem atuam. Na ZDP também ocorrem as interações ou as mediações cognitivas na qual se destaca a ação de um instrutor (ou um parceiro mais capaz) que atua no apoio para a busca dos novos conhecimentos ou ampliação do nível atual de desenvolvimento. $O$ processo de internalização onde, mediante a um ambiente propício de aprendizagem social, se estabelece critérios autônomos e individuais no qual o indivíduo internaliza e retêm o aprendizado externo (JENSEN; KUMMER, 2015).

\title{
2.6 Estratégias de Ensino e Aprendizagem
}

Para Petrucci; Batiston (2006, p. 263), a palavra estratégia esteve, historicamente, vinculada à arte militar no planejamento das ações a serem executadas nas guerras e, atualmente, largamente utilizada no ambiente empresarial. Porém, os autores admitem que:

[...] a palavra 'estratégia' possui estreita ligação com o ensino. Ensinar requer arte por parte do docente, que precisa envolver o aluno e fazer com ele se encante com o saber. O professor precisa promover a curiosidade, a segurança e a criatividade para que o principal objetivo educacional, a aprendizagem do aluno, seja alcançada.

Desse modo, o uso do termo "estratégias de ensino" refere-se aos meios utilizados pelos docentes na articulação do processo de ensino, de acordo com cada atividade e os resultados esperados (ANASTASIOU; ALVES, 2004).

\section{APLICAÇÃO E RESULTADOS}

\subsection{Resultados da aplicação do questionário de Estilo de Aprendizagem}

Para avaliar essas dimensões, Felder; Soloman (1991) desenvolveram, a partir do Modelo de Felder; Silverman, um instrumento chamado ILS (Index of Learning Styles), para identificar os estilos de aprendizagem dos alunos dentro das quatro dimensões propostas. O questionário é composto por 44 questões objetivas, sendo que 11 perguntas são para cada dimensão, como pode ser visto na Tabela 2. 
Tabela 2 - Resultados obtidos com a aplicação do questionário de estilo de aprendizagem

\begin{tabular}{|c|c|c|c|c|c|c|c|c|c|c|c|}
\hline \multicolumn{3}{|c|}{ Ativo/Reflexivo } & \multicolumn{3}{|c|}{ Sensitivo/Intuitivo } & \multicolumn{3}{|c|}{ Visual/Verbal } & \multicolumn{3}{|c|}{ Sequencial/Global } \\
\hline $\mathbf{Q}$ & a & b & $\mathbf{Q}$ & $a$ & b & $\mathbf{Q}$ & $a$ & b & $\mathbf{Q}$ & $a$ & b \\
\hline 1 & 1 & 0 & 2 & 1 & 0 & 3 & 1 & 0 & 4 & 0 & 1 \\
\hline 5 & 1 & 0 & 6 & 1 & 0 & 7 & 1 & 0 & 8 & 1 & 0 \\
\hline 9 & 1 & 0 & 10 & 1 & 0 & 11 & 1 & 0 & 12 & 1 & 0 \\
\hline 13 & 1 & 0 & 14 & 1 & 0 & 15 & 0 & 1 & 16 & 1 & 0 \\
\hline 17 & 0 & 1 & 18 & 1 & 0 & 19 & 1 & 0 & 20 & 1 & 0 \\
\hline 21 & 0 & 1 & 22 & 1 & 0 & 23 & 1 & 0 & 24 & 1 & 0 \\
\hline 25 & 0 & 1 & 26 & 0 & 1 & 27 & 1 & 0 & 28 & 0 & 1 \\
\hline 29 & 1 & 0 & 30 & 1 & 0 & 31 & 1 & 0 & 32 & 1 & 0 \\
\hline 33 & 1 & 0 & 34 & 0 & 1 & 35 & 1 & 0 & 36 & 1 & 0 \\
\hline 37 & 0 & 1 & 38 & 1 & 0 & 39 & 1 & 0 & 40 & 0 & 1 \\
\hline 41 & 1 & 0 & 42 & 1 & 0 & 43 & 1 & 0 & 44 & 1 & 0 \\
\hline \multicolumn{12}{|c|}{ Total (soma X's de cada coluna) } \\
\hline \multicolumn{3}{|c|}{ Ativo/Reflexivo } & \multicolumn{3}{|c|}{ Sensitivo/Intuitivo } & \multicolumn{3}{|c|}{ Visual/Verbal } & \multicolumn{3}{|c|}{ Sequencial/Global } \\
\hline & a & b & & a & b & & a & b & & a & b \\
\hline & 7 & 4 & & 9 & 3 & & 10 & 1 & & 8 & 3 \\
\hline \multicolumn{12}{|c|}{ maior - menor $=$ letra do maior } \\
\hline & $3 a$ & & & 6a & & & 9a & & & $5 a$ & \\
\hline
\end{tabular}

Fonte: Autores

O resultado aponta as dimensões dominantes expressas em três escalas: a) equilibrado, que indica preferência entre ambas as dimensões; b) moderada, que indica preferência moderada pela dimensão dominante; c) forte, indica preferência forte pela dimensão dominante. A predominância em um dos polos de cada dimensão do modelo não exclui a outra, pois ela pode mudar de acordo com o tipo de conteúdo que está sendo apresentado, como também pode mudar com o passar do tempo (CURY, 2000). Na Tabela 3 estão destacados em amarelo os resultados presentes na Tabela 2, considerando as três escalas; por exemplo, o resultado "3a" (coluna Ativo/Reflexivo) encontra-se na escala "Equilibrado".

Tabela 3 - Conversão em escala dos resultados obtidos com o questionário de estilo de aprendizagem

\begin{tabular}{|c|c|c|c|c|c|c|c|c|c|c|c|c|c|}
\hline ATI & & & & & & & & & & & & & REF \\
\hline & 11a & $9 \mathrm{a}$ & $7 \mathrm{a}$ & $5 a$ & $3 a$ & 1a & $1 \mathrm{~b}$ & $3 b$ & $5 b$ & 76 & $9 b$ & $11 \mathrm{~b}$ & \\
\hline$\overline{\mathrm{SEN}}$ & & & & & & & & & & & & & INT \\
\hline & $11 \mathrm{a}$ & $9 \mathrm{a}$ & $7 a$ & $5 a$ & $3 a$ & $1 \mathrm{a}$ & $1 \mathrm{~b}$ & $3 \mathrm{~b}$ & $5 b$ & 76 & $9 \mathrm{~b}$ & $11 \mathrm{~b}$ & \\
\hline VIS & & & & & & & & & & & & & VER \\
\hline & $11 \mathrm{a}$ & $9 \mathrm{a}$ & $7 a$ & $5 \mathrm{a}$ & $3 a$ & $1 \mathrm{a}$ & $1 \mathrm{~b}$ & $3 \mathrm{~b}$ & $5 b$ & 76 & $9 \mathrm{~b}$ & $11 \mathrm{~b}$ & \\
\hline SEQ & & & & & & & & & & & & & GLO \\
\hline & $11 \mathrm{a}$ & $9 \mathrm{a}$ & $7 \mathrm{a}$ & $5 a$ & $3 a$ & $1 \mathrm{a}$ & $1 \mathrm{~b}$ & $3 b$ & $5 \mathrm{~b}$ & $7 b$ & $9 \mathrm{~b}$ & $11 \mathrm{~b}$ & \\
\hline & & orte & & derado & & & ilbrad & & & derado & & Forte & \\
\hline
\end{tabular}

ATI (ativo); REF (reflexivo); SEN (sensitivo); INT (intuitivo); VIS (visual); VER (verbal); SEQ (sequencial); GLO (global). Fonte: Autores

Os resultados obtidos pelos questionários aplicados demonstram que os alunos têm uma tendência aos estilos de aprendizagem nas dimensões ativa, sensitiva, visual (maior destaque), e sequencial, o que reforça a questão da definição das estratégias de ensino e aprendizagem que precisam ter como alicerce uma linha ou teoria de aprendizagem que neste caso apontam para Vygotsky, que professa as questões relativas a mediação pelo parceiro mais capaz relacionada à questão do aprendizado ativo no qual os alunos tendem a trabalhos em grupo, o parceiro mais capaz neste caso passam a ser os colegas e professor trabalhando de forma cooperativa para a construção do conhecimento. Os sistemas simbólicos por meio de símbolos e signos operam na articulação do saber com base no referencial sociocultural dos discentes, por meio de imagens, gráficos, infográficos, textos, vídeos e demais recursos que apontam para as necessidades visuais 
dos mesmos (dimensão visual). Com relação tendências sensitiva e sequencial a abordagem utilizada no conceito da Zona de Desenvolvimento Proximal que parte de conhecimentos práticos, isto é, da realidade do aluno para o desenvolvimento do conhecimento concreto ou proximal, que vai ao encontro da necessidade de uma construção lógica e linear dos saberes como apontam estes estilos de aprendizagem.

\subsection{Aplicação da AHP (Analytic Hierarchy Process)}

A função da aplicação da AHP e seria criar uma hierarquia de prioridade entre as abordagens de ensino a aprendizagem propostas para este projeto. A hierarquia de prioridades resultante da aplicação da ferramenta pode ser vista na Tabela 4, na qual estão tabulados os resultados da análise AHP.

Tabela 4 - Hierarquia das abordagens de ensino e aprendizagem.

\begin{tabular}{c|c|c|c|c|c}
\hline & ATIVO & SENSITIVO & VISUAL & SEQUENCIAL & $\begin{array}{c}\text { VETOR DE } \\
\text { DECISÃO }\end{array}$ \\
\hline VETOR DE CRITÉRIO & $6 \%$ & $26 \%$ & $56 \%$ & $12 \%$ & \\
\hline $\begin{array}{c}\text { SALA DE AULA } \\
\text { INTERVERTIDA }\end{array}$ & $20 \%$ & $31 \%$ & $23 \%$ & $6 \%$ & $23 \%$ \\
\hline $\begin{array}{c}\text { APRENDIZAGEM BASEADA } \\
\text { EM TIMES }\end{array}$ & $7 \%$ & $5 \%$ & $6 \%$ & $30 \%$ & $9 \%$ \\
\hline $\begin{array}{c}\text { APRENDIZAGEM BASEADA } \\
\text { EM PROJETOS }\end{array}$ & $20 \%$ & $22 \%$ & $24 \%$ & $21 \%$ & $23 \%$ \\
\hline $\begin{array}{c}\text { INSTRUÇÃO POR PARES } \\
\text { SOCIOINTERACIONISMOS } \\
\text { (Vygotsky) }\end{array}$ & $7 \%$ & $11 \%$ & $7 \%$ & $30 \%$ & $11 \%$ \\
\hline
\end{tabular}

Fonte: Autores

Os resultados apontam para a predominância de uma abordagem sociointeracionista em detrimento das demais, este fato se deve pela abrangência de possibilidades de estratégias educacionais que a mesma permite realizar em um ambiente de ensino. Outro fato relevante é a necessidade de esclarecer que praticamente todos os métodos ativos usados como base para este estudo têm certa relação com cognitivismo. É oportuno salientar que não existe método melhor ou pior, mas aquele mais adequado ao que se pretende ensinar e, se existe um método inadequado, este é o autoritário.

\subsection{Estrutura para roteiro de aula}

Com base em todos nos resultados obtidos e suas interpretações relativas ao instrumento ILS demonstrando a tendência para as abordagens ativa, sensitiva, visual e sequencial e o apontamento da AHP ao sociointeracionista de Vygotsky, o qual trabalha com as questões relativas à ZDP, as mediações cognitivas e o processo de interiorização do conhecimento, como forma de atingir os objetivos propostos por este projeto. A estrutura para organização das aulas adotada após os estudos realizados é apresentada na Figura 2. 
Figura 2 - Modelo exemplificado para organização das estratégias de ensino e aprendizado

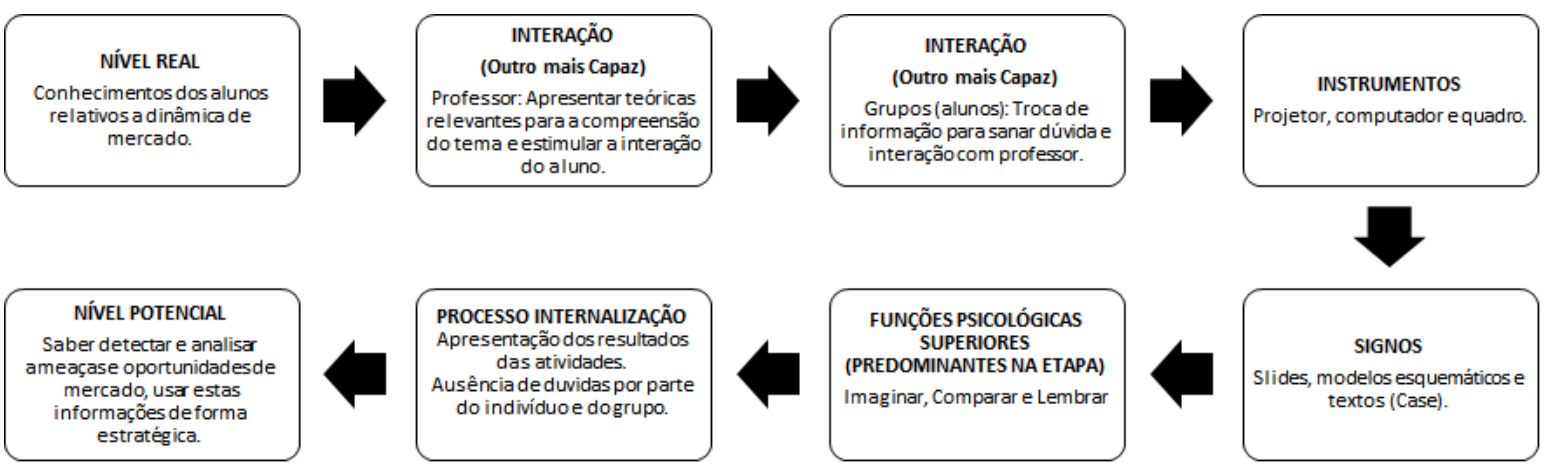

Fonte: Autores

\section{CONSIDERAÇÕES FINAIS}

O atual cenário do ensino superior e o perfil do jovem reafirmam a necessidade de um novo olhar do professor para o enfrentamento destes desafios em sua profissão. Mas é preciso compreender o que significa este "novo olhar", pois para muitos é apenas a busca de abordagens prontas que supostamente irão favorecer ao aprendizado dos alunos por oferecer características mais voltadas a prática, mas que por muitas vezes precisam ser adaptadas ou trabalhadas do ponto de vista didático-pedagógico para atender às reais necessidades destes educandos, que se modificam de turma para turma.

Os resultados obtidos até o momento demonstram que os estudos das teorias de aprendizagem ofereceram modelos que visam explicar os processos de aprendizagem dos indivíduos com base na psicologia e educação o que levou a compreensão que o sociointeracionismo de Vygotsky apresenta os elementos necessários para a orientação deste estudo e para uma elaboração de proposta de estrutura organizacional das aulas. O questionário de estilo de aprendizagem por meio das tendências dos alunos as preferências de aprendizagem orientadas para as dimensões ativo, sensitivo, visual e sequencial, serviu como balizador para a construção das abordagens utilizadas e o uso de ferramentas da engenharia com o objetivo de orientar e validar de forma lógica as tomadas de decisão analisando as variáveis presentes nos problemas estudados, o alinhamento destes processos apresentaram resultados que viabilizaram a criação de estratégias que favorecem aos alunos na aquisição do conhecimento. Ainda será aplicado neste projeto, em uma próxima etapa, o diagrama de Kano para validar a percepção dos alunos com relação as estratégias propostas possibilitando a implementação de melhoria neste processo.

A presente pesquisa, em andamento, já demonstra a importância da adoção de um referencial teórico educacional na elaboração das atividades pedagógicas, respeitando-se os processos cognitivos e os estilos de aprendizagem dos envolvidos, favorecendo a obtenção e retenção do conhecimento (competências) e a capacidade de realização de ações fundamentadas no conhecimento adquirido (habilidades). Também sinaliza as formas preferenciais de aprendizagem dos estudantes de engenharia estudados, principalmente a percepção visual, as quais devem fazer parte do planejamento das aulas, visando uma maior eficácia no ambiente de ensino.

\section{BIBLIOGRÁFIA}

ANASTASIOU, Léa das Graças Camargos; ALVES, Leonir Pessate. Estratégias de ensinagem. In: ANASTASIOU, Léa das Graças Camargos; ALVES, Leonir Pessate. (Orgs.). 
Processos de ensinagem na universidade. Pressupostos para as estratégias de trabalho em aula. 3. ed. Joinville: Univille, 2004. p. 67-100.

BRUNER, J. (1997b). Celebrating divergence: Piaget and Vygotsky. Journal of Human Development, 40, 63-73.

CURY, H.N. Estilos de Aprendizagem de Alunos de Engenharia. Anais do XXVII Congresso Brasileiro de Ensino de Engenharia. 2000. Ouro Preto, MG.

ERTMER, P. A.; NEWBY, T. J. Behaviorism, Cognitivism, Constructivism: Comparing Critical Features From an Instructional Design Perspective. Performance Improvement Quarterly, v. 26, n. 2, p. 43-71, 2013a.

FELDER, R. M.; SILVERMAN, L. K. Learning and Teaching Styles in Engineering Education. Journal of Engineering Education, n. January 1988, p. 674-681, 2002.

FELDER, R. M.; SOLOMAN, B. A. Index of learning styles questionnaire. North Carolina State University, 1991. Disponível em: 〈http://www.ncsu.edu/felder-public/ILSdir.html>. Acesso em: 12 dezembro 2018.

FELDER, R. M.; SPURLIN, J. Applications, reliability and validity of the index of learning stylesInternational Journal of Engineering Education, 2005.

FLEMING, N. D. Teaching and learning styles: VARK strategies. Christchurch, New Zealand: N. D. Fleming, 2001.

FERREIRA, J. B.; SADOYAMA, A. S. P.; CORREIA, A. F. C.; GOMES, P. A. T.P. Diversidade e gênero no contexto organizacional: um estudo bibliometrico. Revista Pensamento Contemporâneo, Rio de Janeiro, v. 9, n. 3, p. 45-66, jul./set. 2015.

GIL, A. C. Como elaborar projetos de pesquisa. 5. ed. São Paulo: Atlas, 2010.

JENSEN, J. L.; KUMMER, T. A.; D M GODOY, P. D. Improvements from a flipped classroom may simply be the fruits of active learning. CBE life sciences education, United States, v. 14, n. 1, p. 5, 2 mar. 2015.

LAI, W.; LIN, C. Constructing business incubation service capabilities for tenants at postentrepreneurial phase. Journal os Business Research, v. 68, n. 11, p. 2285-2289, 2015. Disponível em: <http://sciencedirect.com/science/article/pii/S0148296315002404> Acesso em: 12 set. 2018.

LEFRANÇOIS, Guy R. Teorias da aprendizagem: o que o professor disse. 2. ed. São Paulo: Cengage, 2017. $475 \mathrm{p}$.

MAZZIONI, S. As estratégias utilizadas no processo de ensino-aprendizagem: concepções de alunos e professores de ciências contábeis. Revista Eletrônica de Administração e Turismo ReAT. v. 2, n. 1, jan./jun., 2013. p. 93-109. Disponível em: Acesso em: 22 nov. 2014.

MOREIRA, Marco Antonio. Teorias de aprendizagem. $2^{\text {a }}$ Ed. Ampl., São Paulo, E.P.U., 2017.

NUNES, A. I. B. L; SILVEIRA, R. N. Psicologia da aprendizagem: processos, teorias e contextos. $2^{a}$ Ed. Brasília. Liber livro. 2009.189

OLIVEIRA, S. Geração Y: era das conexões, tempo dos relacionamentos. São Paulo: Clube de Autores, 2009. 
PETRUCCI, V. B. C.; BATISTON, R. R.. Estratégias de ensino e avaliação de aprendizagem em contabilidade. In: PELEIAS, Ivan Ricardo. (Org.) Didática do ensino da contabilidade. São Paulo: Saraiva, 2006.

RAMMAZZINA FILHO, W. A.; BATISTA, I.; LORENCINI, A. Formação de professores de engenharia - desafios e perspectivas. In: SIMPÓSIO DE ENSINO DE CIÊNCIA E TECNOLOGIA. Anais eletrônicos. Ponta Grossa: [s. n.], p. 1-10, 2014.

SAATY, T. L; TRAIN, L. T. On the invalidity of fuzzifying numeral judments in the Analytic Hierarchy Process. Mathematical and Computer Modelling, v. 46, n 7, p.962-975, 2007.

SAATY, T. L.; VARGAS, L. G. The analytic hierarchy process: Wash criteria should not be ignored. International Journal of Management and Decision Making, v, 7, n 2-3, p. 180-188, 2006.

STAVENGA JONG, J. A.; WIERSTRA, R. F. A.; HERMANUSSEN, J. An exploration of the relationship between academic and experiential learning approaches in vocational education. British Journal of Educational Psychology, v. 76, n. 1, p. 155-169, 2006.

UNESCO, Nações Unidas Brasil. Número de universitários dobrou no mundo entre 2000 e 2014, 2017. Disponível em: <https://nacoesunidas.org/numero-de-universitarios-dobrou-nomundo-entre-2000-e-2014-diz-unesco/> Acesso em: 20 de nov. 2018

VYGOTSKY, L. S. Mind in Society: Development of Higher Psychological Processes. $1^{\mathrm{a}}$ edition ed. London, United Kingdom: Harvard University Press, 1978. v. 1

\title{
ANALYSIS OR NEEDS FOR THE PREPARATION OF TEACHING AND LEARNING STRATEGIES FOR PRODUCTIO ENGINEERING IN THE MARKETING DISCIPLINE.
}

\begin{abstract}
This paper presents an action research about possible ways of teaching and learning applied to the Production Engineering course. The research and the method were developed and applied to fourth-year Production Engineering students in Marketing discipline, and it's worth to emphasize that the researches were submitted and approved by an ethic committee and it's registered in "Plataforma Brasil". The objective of this research consists in developing educational approaches that meet the involved students' needs, aiming to have the most suitable approaches for their learning style. The defined strategies were based on the Index of Learning Styles (ILS) questionnaires application, that evaluate preferences on four dimensions of the learning style model, offering insights about teaching and learning, based on educational psychology and on engineering teaching; the Analytic Hierarchy Process (AHP) was also used with the intention of creating a priorities hierarchical scale of the information gotten from the surveys concerning the analyzed methods for the strategies definition, based on Vygotsky's social interactionist education theoretical referential. The results obtained so far point to the matter of the necessity of defining in a scientific way the means of teaching and learning that serve the students in question; these strategies were already properly defined and they are in the process of being implemented, and an evaluation of students' perception regarding the applied strategies for the verification of possible improvements is suitable for a second phase, for this purpose it's intended to apply the Kano method or diagram.
\end{abstract}

Keywords: Strategy. Teaching and Learning. Vygotsky. Engineering. 